Bundesgesundheitsbl 2017 · 60:697-706 DOI 10.1007/s00103-017-2563-4

Online publiziert: 1. Juni 2017

(c) Der/die Autor(en) 2017. Dieser Artikel ist eine Open-Access-Publikation.

CrossMark

Ulrike Pabel · Thorsten Buhrke - Klaus Abraham • Thilo Nölke • Matthias Gehling • Alfonso Lampen · Monika Lahrssen-Wiederholt · Reiner Wittkowski

Bundesinstitut für Risikobewertung, Berlin, Deutschland

\title{
Persistente organische
} Kontaminanten in Lebensmitteln

\section{Exposition, Gefährdungspotenzial und gesundheitliche Bewertung}

Eine Reihe von organischen Verbindungen zeichnet sich durch eine hohe chemische und thermische Stabilität aus und ist damit biologisch nur schlecht abbaubar. Dies führt zu einer globalen Verteilung dieser Verbindungen in Böden, Gewässern und Luft und letztlich zum Eintrag in die Nahrungskette. Diese sogenannten persistenten organischen Kontaminanten entstehen entweder durch technische und natürliche Verbrennungsprozesse oder sie werden aufgrund ihrer besonderen Materialeigenschaften gezielt zur Anwendung in einer Vielzahl von Produkten hergestellt. Der Beitrag befasst sich mit der gesundheitlichen Bewertung einer Auswahl von 3 Stoffgruppen persistenter organischer Kontaminanten:

- Per- und Polyfluoralkylsubstanzen (PFAS),

- polychlorierte Dibenzo- $p$-dioxine (PCDD), Dibenzofurane (PCDF) und polychlorierte Biphenyle (PCB) sowie

- polyzyklische aromatische Kohlenwasserstoffe (PAK), für die Lebensmittel einen Hauptpfad der menschlichen Exposition darstellen.

Die Problematik der Emissionen von $\mathrm{PCDD} / \mathrm{F}$ wurde bereits in den 1970erJahren erkannt und durch Regulierungsmaßnahmen soweit eingedämmt, dass in den vergangenen 30 Jahren ein deutlicher Rückgang der Exposition verzeichnet werden konnte. PCDD/F und PCB gehörten zu den ersten 12 organischen Chlorverbindungen, bekannt als „dirty dozen“, die in der Stockholmer
Konvention über persistente organische Kontaminanten (POP-Konvention) weltweit geregelt wurden. Die Bedeutung von PFAS, die seit den 1950er-Jahren industriell produziert werden, als globale anthropogene Umweltkontaminanten wurde hingegen erst viel später erkannt. Vor 10 Jahren wurden Regulierungsmaßnahmen eingeleitet, die sich jedoch bislang nur auf wenige Verbindungen dieser Stoffgruppe beziehen.

Die gesundheitliche Bewertung jeder dieser 3 Stoffgruppen bringt die Herausforderung mit sich, dass für Menschen in der Regel eine Exposition gegenüber komplexen und variablen Mischungen mit unterschiedlichen Profilen der vorkommenden Einzelverbindungen einer Stoffgruppe und ihrer jeweiligen Mengenanteile im Gemisch besteht. Bei der Risikobewertung stehen verschiedene toxikologische Endpunkte für die jeweiligen Stoffgruppen im Vordergrund, wobei der Kenntnisstand zu den molekularen Mechanismen der toxischen Wirkungen unterschiedlich hoch ist. Dies bedingt verschiedene Konzepte für die gesundheitliche Bewertung der Exposition gegenüber den Stoffgemischen der organischen Kontaminanten, die im Folgenden skizziert werden.

\section{Per- und Polyfluoralkyl- substanzen}

PFAS sind eine Gruppe von Industriechemikalien, die für die Herstellung von Oberflächenbeschichtungen mit wasser-, fett- und schmutzabweisenden Eigenschaften verwendet werden. Sie finden sich in einer Vielzahl von Gebrauchsgegenständen, so z. B. in Beschichtungen von Kochgeschirr und Textilien (Outdoor-Kleidung, Imprägnierungen von Auslegware und Möbelbezügen) und in Produkten der Papierindustrie (Backpapier, Kartons mit Antihaftbeschichtung, Hochglanzpapier). Auch weitere Produkte, wie z. B. Skiwachse, Imprägniersprays und Feuerlöschschäume können PFAS enthalten. Im Großteil dieser Produkte liegen PFAS in Polymeren fest gebunden vor, sodass man zunächst davon ausging, dass für Personen, die nicht berufsbedingt gegenüber PFAS exponiert sind, nur eine marginale Exposition besteht. In den vergangenen Jahrzehnten haben sich PFAS dennoch zu globalen Kontaminanten in Wasser, Böden und Luft entwickelt und sind auch in menschlichen Blutproben nachweisbar [1]. Hierfür ist die außerordentliche Stabilität der PFAS verantwortlich, die in der Chemie der starken kovalenten Kohlenstoff-Fluor-Bindung begründet ist und die gleichzeitig für die einzigartigen Materialeigenschaften, die von PFAS vermittelt werden, verantwortlich ist. Für diese Substanzgruppe gibt es daher bezüglich ihrer Persistenz kaum Vergleichbares und selbst ein marginaler Eintrag in die Umwelt führt über einen größeren Zeitraum in Verbindung mit der Mobilität der Stoffe zu ihrer globalen Verbreitung. Verstärkte Aufmerksamkeit schenkt man den Stoffen, seitdem bekannt wurde, dass in bestimmten Regionen, auch in Deutschland, PFAS durch die Ausbringung von Klärschlämmen bzw. Rückständen aus 


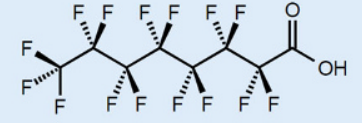

Perfluoroktansäure (PFOA)

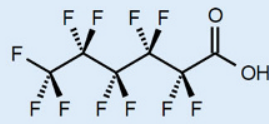

Perfluorhexansäure (PFHxA)<smiles>O=C(O)C(F)(F)C(F)(F)C(F)(F)F</smiles>

Perfluorbutansäure (PFBA)<smiles>O=C(O)C(F)(F)[C@@H](F)OC(F)(F)C(F)(F)C(F)(F)OC(F)(F)F</smiles>

4,8-Dioxa-3H-perfluornonansäure (Adona)

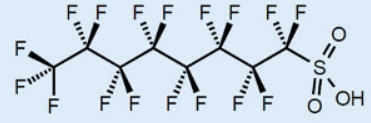

Perfluoroktansulfonsäure (PFOS)

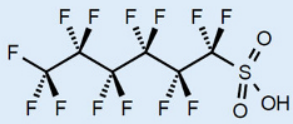

Perfluorhexansulfonsäure (PFHxS)

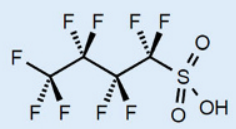

Perfluorbutansulfonsäure (PFBS)

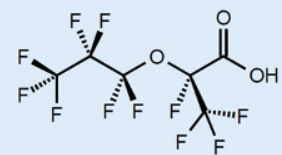

2,3,3,3-Tetrafluoro-2(heptafluoropropoxy) propansäure (GenX)
Abb. $1<$ Strukturen ausgewählter Per-und Polyfluoralkylsubstanzen der Papierindustrie in Ackerflächen eingetragen wurden und sich daraufhin erhöhte Gehalte in Umweltmedien, Trinkwasser und Lebensmitteln fanden [2].

Die Leitsubstanzen der PFAS sind die C8-Verbindungen Perfluoroktansäure (PFOA) und Perfluoroktansulfonsäure (PFOS); (@ Abb. 1). Aufgrund ihrer persistenten und reproduktionstoxischen Eigenschaften wurde PFOS im Jahr 2009 in den Annex B der POPKonvention aufgenommen; seitdem ist die Verwendung von PFOS und ihren Derivaten beschränkt. PFOA sowie die perfluorierten Säuren mit einer Kohlenstoffkettenlänge von C9 bis C14 wurden in die europäische Kandidatenliste der besonders besorgniserregenden Stoffe (SVHC) aufgenommen.

Aufgrund dieser Beschränkungen ist die Industrie gezwungen, für ihre Anwendungen auf alternative Verbindungen auszuweichen. Dies sind u. a. kürzerkettige Homologe, wie z. B. Perfluorhexansäure (PFHxA) oder Perfluorbutansulfonsäure (PFBS) und Derivate wie z. B. GenX oder Adona, bei denen die fluorierte Kohlenstoffkette durch Sauerstoffatome unterbrochen ist (• Abb. 1).
Letztendlich handelt es sich bei all diesen Substituten aber auch um perfluorierte Verbindungen, die im Vergleich zu PFOA und PFOS aus toxikologischer Sicht zwar weniger bedenklich erscheinen, deren Einsatz aufgrund der vergleichbaren Persistenz in der Umwelt jedoch ebenfalls Grund zur Besorgnis gibt. Die Regulation kurzkettiger PFAS im Rahmen der Europäischen Chemikalienverordnung gestaltet sich u. a. deshalb schwierig, weil die Stoffe zum Teil nicht als solche zur Anwendung in Verbraucherprodukten hergestellt werden und daher nicht registriert werden müssen, sondern entweder im Herstellungsprozess anderer PFAS eine Rolle spielen oder erst durch Biotransformation von sog. Vorläuferstoffen entstehen. Die Gruppe der PFAS umfasst neben Perfluoralkylsäuren (PFAA) mit kürzeren und längeren perfluorierten Kohlenstoffketten auch Fluortelomeralkohole und Polymere [3] sowie Vorläuferstoffe wie Fluortelomerphosphatester, -acrylate und -iodide sowie Perfluoralkylsulfonamide, die nach Biotransformation zu PFAA indirekt zur Exposition gegenüber PFAA beitragen können [4].<smiles>Clc1ccc2c(c1)Oc1ccc(Cl)cc1O2</smiles><smiles>O=[PH](=O)(Cl)c1ccc2c(c1)oc1ccc(Cl)cc12</smiles>

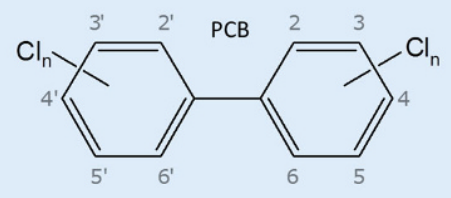

Abb. 2 A Strukturformeln der polychlorierten Dibenzo- $p$-dioxine $(P C D D)$, Dibenzofurane $(P C D F)$ und polychlorierten Biphenyle $(P C B)$. An die Benzolringe der PCDD/F können bis zu 8 Chloratome, an PCB bis zu 10 Chloratome gebunden sein. Die Toxizität der Verbindungen ist abhängig von der Anzahl und der Stellung der Chloratome

\section{Gefährdungspotenzial}

PFAS werden gut resorbiert und reichern sich im Blutserum an, wo sie unspezifisch an Serumproteine wie z. B. Serumalbumin binden. Die Ausscheidung erfolgt über die Niere, wobei die Ausscheidung beim Menschen deutlich langsamer erfolgt als bei anderen Spezies (• Tab. 1). Die Halbwertzeiten für PFOA und PFOS im Blut liegen beim Menschen im Bereich von 4 bis 6 Jahren. Hinsichtlich der Ausscheidung sind die kürzerkettigen Derivate im Vorteil; so beträgt die Halbwertzeit von z. B. PFHxA oder PFBS beim Menschen etwa einen Monat (• Tab. 1).

Hinsichtlich ihrer Toxizität sind vor allem PFOA und PFOS gut charakterisiert. Beide Substanzen zeigen eine geringe akute Toxizität, in subchronischen und chronischen Studien wurden jedoch diverse toxische Effekte mit zum Teil steilen Dosis-Wirkungs-Kurven beobachtet. Die wiederholte Gabe von PFOA bzw. PFOS führte im Tierversuch bei verschiedenen Spezies primär zu adversen Effekten in der Leber (hepatozelluläre Hypertrophie, Vakuolisierung) und der Schilddrüse sowie zu Veränderungen bei den Blutlipidspiegeln. In 
Bundesgesundheitsbl 2017·60:697-706 DOI 10.1007/s00103-017-2563-4

(c) Der/die Autor(en) 2017. Dieser Artikel ist eine Open-Access-Publikation.

U. Pabel · T. Buhrke · K. Abraham · T. Nölke · M. Gehling · A. Lampen · M. Lahrssen-Wiederholt · R. Wittkowski

\section{Persistente organische Kontaminanten in Lebensmitteln. Exposition, Gefährdungspotenzial und gesundheitliche Bewertung}

\section{Zusammenfassung}

Sowohl natürliche als auch technische Verbrennungsprozesse sowie industrielle Herstellungsprozesse und die Freisetzung aus Produkten führen zum Eintrag von organischen Kontaminanten in die Umwelt. Für einige dieser Stoffe stellen Lebensmittel einen Hauptpfad der menschlichen Exposition dar. Dazu gehören 3 Stoffgruppen persistenter organischer Kontaminanten: (1) Per- und Polyfluoralkylsubstanzen (PFAS), (2) polychlorierte Dibenzo- $p$-dioxine, Dibenzofurane (PCDD/F) und polychlorierte Biphenyle (PCB) sowie (3) polyzyklische aromatische Kohlenwasserstoffe (PAK). Während die Problematik der Emissionen von PCDD/F bereits in den 1970er-Jahren erkannt und mittlerweile effektiv reguliert worden ist, wurde die Bedeutung von PFAS als globale anthropogene Umweltkontaminanten erst deutlich später erkannt.

Zur Erfassung der Höhe des toxischen Potenzials einer Mischexposition ist für PCDD/F und bestimmte PCB ein Toxizitätsäquivalenzfaktor(TEF)-System etabliert. Für die Stoffgruppen PFAS bzw. PAK wird ein solches System bislang nicht für die gesundheitliche Bewertung und die Regulation herangezogen. Aktuell wird für PFAS die Reevaluierung der bisherigen gesundheitlichen Leitwerte diskutiert, da mittlerweile neue Erkenntnisse zur Toxikologie und Epidemiologie vorliegen.
Die Persistenz der diskutierten Stoffgruppen in der Umwelt führt dazu, dass ein Eintrag in die Lebensmittelkette über lange Zeiträume bestehen bleibt, auch wenn Umwelteinträge minimiert werden. Dies erfordert eine langfristige Weiterführung des Monitorings von Lebensmitteln sowie die vorausschauende Weiterentwicklung von Risikobewertungsansätzen und Regulationsmaßnahmen.

\section{Schlüsselwörter}

Persistent $\cdot$ Polychlorierte Dibenzo- $p$-dioxine und Dibenzofurane · Polychlorierte Biphenyle Per- und Polyfluoralkylsubstanzen · Polyzyklische aromatische Kohlenwasserstoffe

\section{Persistent organic contaminants in food. Exposure, hazard potential, and health assessment}

\section{Abstract}

Environmental emissions of organic contaminants are caused by man-made and natural combustion processes, industrial production facilities, and the release from products. Food represents the main source of human exposure for some of these compounds. This is the case for three groups of persistent organic contaminants: (1) per- and polyfluoroalkyl substances (PFAS), (2) polychlorinated dibenzo- $p$-dioxins (PCDDs), polychlorinated dibenzofurans (PCDFs), polychlorinated biphenyls (PCBs), and for (3) polycyclic aromatic hydrocarbons (PAHs). The issues regarding $\mathrm{PCDD} / \mathrm{F}$ emissions were already recognized as a problem in the 1970s and have since then been effectively regulated; the impact of PFAS as global anthropogenic environmental contaminants was identified much later.

A system of toxicity equivalency factors (TEF system) was established for the assessment of the toxic potency of a mixed exposure to $P C D D / F$ and certain PCBs. For the health assessment and regulation of PAHs and PFAS, no such system has been implemented so far. For PFAS, a re-evaluation of the present tolerable daily intake values (TDI values) is currently being discussed, as new insights into toxicology and epidemiology have been gained.
The persistence in the environment of the compound groups discussed here leads to entry into the food chain over long periods of time, even if access into the environment is minimized. This requires a long-term continuation of the monitoring of food stuffs and forward-looking risk assessment approaches and regulatory measures.

\section{Keywords}

Persistent · Polychlorinated dibenzo- $p$-dioxins and dibenzofurans . Polychlorinated biphenyls · Per- and polyfluoroalkyl substances . Polycyclic aromatic hydrocarbons einer 2-Jahres-Studie führte die wiederholte orale Gabe von PFOS bei Ratten $\mathrm{zu}$ einer erhöhten Inzidenz von Adenomen in Leber und Schilddrüse, bei PFOA wurden in einer vergleichbaren Studie vermehrt Adenome in Leber, Pankreas und Hoden festgestellt. Beide Substanzen werden als nicht genotoxische Kanzerogene bewertet. Außerdem zeigen PFOA und PFOS immuntoxische und reproduktionstoxische Effekte im Tierversuch $[8,9]$. Die wiederholte orale Gabe von PFOS führte bei trächtigen Ratten zu einer stark verminderten Anzahl lebensfähiger Nachkommen sowie $\mathrm{zu}$ einer verzögerten Entwicklung der Lebendgeborenen. Im Fall von PFOA wurden vergleichbare entwicklungstoxische Effekte beobachtet. Bei Mäusen war unter PFOA-Behandlung vor allem die ungewöhnliche Resorption sämtlicher Embryonen oder Föten eines Wurfes während der Schwangerschaft („full litter resorptions") auffällig [10]. Auch in epidemiologischen Studien, die zum Teil an sehr großen Kohorten durchgeführt wurden, die über das Trinkwasser gegenüber PFAS exponiert waren, werden Zusammenhänge zwischen der Höhe der Gehalte der Stoffe im menschli- chen Körper und der Fertilität, den Geburtsgewichten von Neugeborenen, dem Fettstoffwechsel, den Schilddrüsenhormonen, dem Immunsystem und der hormonellen Entwicklung berichtet [8, 11, 12].

Gesundheitsbezogene Leitwerte, beispielsweise für die tolerierbare tägliche Aufnahme (TDI), wurden von internationalen Gremien bisher nur für PFOS und PFOA abgeleitet. Der TDI gibt die tägliche Dosis an, die bei lebenslanger Aufnahme keine gesundheitlichen Wirkungen beim Menschen erwarten lässt. In Abhängigkeit davon, welcher Ansatz 
Tab. 1 Halbwertzeiten ${ }^{\mathrm{a}}$ von Per- und Polyfluoralkylsubstanzen in Blut bei verschiedenen Spezies (Ergänzt nach [5])

\begin{tabular}{|c|c|c|c|c|c|c|c|c|}
\hline \multirow[t]{2}{*}{ Spezies } & \multicolumn{3}{|c|}{ Perfluorsulfonsäuren } & \multicolumn{5}{|c|}{ Perfluorcarbonsäuren } \\
\hline & PFBS & PFHxS & PFOS & PFBA & PFHxA & PFHpA & PFOA & PFNA \\
\hline Ratte & $4,0 h$ & $29 d$ & $62-71 d$ & $1,0-1,8 h$ & $0,4-0,6 h$ & - & $2-4 h$ & $1,4 d$ \\
\hline Maus & - & $25-27 d$ & $31-38 d$ & $3 h$ & $\sim 1,2 \mathrm{~h}$ & - & $17 d$ & $26-68 d$ \\
\hline Affe & $3,5 \mathrm{~d}$ & $87 d$ & $110 \mathrm{~d}$ & $1,7 \mathrm{~d}$ & $2,4-19,2 h$ & - & $30 d$ & - \\
\hline Schwein & $43 d$ & $2 \mathrm{a}$ & $1,7 \mathrm{a}$ & - & $4,1 d$ & $74 d$ & $236 d$ & - \\
\hline Mensch & $28 d$ & 8,5 a & $5,4 a$ & $3 d$ & $32,0 d$ & $1,2-1,5$ a & $2,3-3,8 a$ & $2,5-4,3 a$ \\
\hline Literatur & {$[5,6]$} & {$[5,6]$} & {$[5,6]$} & [5] & {$[5,6]$} & {$[6,7]$} & {$[5,6]$} & {$[5,7]$} \\
\hline \multicolumn{9}{|c|}{$\begin{array}{l}\text { PFBA Perfluorbutansäure, PFBS Perfluorbutansulfonsäure, PFHpA Perfluorheptansäure, PFHxA Perfluorhexansäure, PFHxS Perfluorhexansulfonsäure } \\
\text { PFNA Perfluornonansäure, PFOA Perfluoroktansäure, PFOS Perfluorsulfonsäure } \\
\text { h Stunden (kursiv), } d \text { Tage, a Jahre (fett), - keine Daten } \\
\text { a Halbwertzeiten weiblicher Tiere, wenn unterschiedliche Halbwertzeiten für die Geschlechter beschrieben sind }\end{array}$} \\
\hline
\end{tabular}

zum Umgang mit den toxikokinetischen Speziesunterschieden gewählt wird und ob die toxikologische Bewertung auf Ergebnissen aus Tierversuchen oder auf epidemiologischen Studien basiert, fallen die Resultate der Ableitungen unterschiedlich aus. Die Europäische Lebensmittelsicherheitsbehörde (EFSA) publizierte im Jahr 2008 TDI-Werte von $0,15 \mu \mathrm{g} / \mathrm{kg}$ Körpergewicht (KG) pro Tag für PFOS und $1,5 \mu \mathrm{g} / \mathrm{kg}$ KG pro Tag für PFOA basierend auf Ergebnissen aus Tierversuchen [13]. Andere Gremien leiteten später unter Verwendung anderer toxikokinetischer Modelle [8, $12,14,15]$ deutlich niedrigere gesundheitsbezogene Leitwerte für PFOS (z. B. $0,03 \mu \mathrm{g} / \mathrm{kg}$ KG pro Tag; [8]) und PFOA (z. B. $0,02 \mu \mathrm{g} / \mathrm{kg}$ KG pro Tag; [8]) ab. Basierend auf epidemiologischen Studien abgeleitete Beurteilungswerte für PFOS und PFOA im Blutplasma liegen bei $2 \mu \mathrm{g}$ PFOA/l und $5 \mu \mathrm{g}$ PFOS/l [12]. Auch die gesundheitliche Bewertung durch die EFSA aus dem Jahr 2008 [13] befindet sich derzeit in Überarbeitung, die voraussichtlich im Jahr 2017 abgeschlossen wird.

Verbraucher sind in der Regel einer Exposition gegenüber verschiedenen PFAS ausgesetzt, die zum Teil ähnliche Wirkungen bzw. Wirkmechanismen besitzen. Eine gesundheitliche Bewertung der Mischexposition wäre daher wünschenswert, wurde aber aufgrund der Vielfalt der infrage kommenden Verbindungen, toxikologischen Endpunkte und der Wirkmechanismen bisher in keinem Bewertungsansatz realisiert.

Auf molekularer Ebene werden die lebertoxischen Effekte von PFOA und
PFOS in erster Linie mit einer Aktivierung des Peroxisom-Proliferatoraktivierten Rezeptors alpha (PPARa) erklärt. PPARa ist ein Transkriptionsfaktor, der in der Leber vor allem an der Regulation des Fettsäuremetabolismus beteiligt ist. Es ist bekannt, dass PPARaAgonisten in Nagern zu den oben genannten lebertoxischen Effekten führen, wohingegen die menschliche Leber weitaus weniger empfindlich gegenüber PPARa-Agonisten reagiert, sodass die PFOA-/PFOS-vermittelte PPARa-Aktivierung nicht als humanrelevant angesehen wird. Verschiedene Studien belegen jedoch, dass diese Substanzen nicht nur PPAR $\alpha$ sondern auch weitere wichtige Transkriptionsfaktoren stimulieren, so z. B. PPAR $\gamma$, der analog zu PPARa den Fettsäuremetabolismus in Fettgewebe reguliert, oder die beiden Transkriptionsfaktoren Pregnan-X-Rezeptor (PXR) und konstitutiver Androstan-Rezeptor (CAR), die am Fremdstoffmetabolismus beteiligt sind [16]. Jüngere Studien zeigen, dass PFOA und PFOS die Aktivität des Hepatozyten-nukleären Faktors 4 alpha (HNF4 $\alpha$ ) inhibieren [17]. HNF4 $\alpha$ ist ein wichtiger Transkriptionsfaktor, der an der Regulation von etwa $40 \%$ aller Gene in der Leber beteiligt ist. Für PFOA und PFOS werden darüber hinaus auch endokrine Effekte diskutiert, die für die beobachtete Reproduktions- und Entwicklungstoxizität der Substanzen ursächlich sein könnten. Verschiedene Studien belegen eine Interaktion von PFOA und PFOS mit den Steroidhormonrezeptoren Estrogenrezeptor alpha $(\mathrm{ER} \alpha)$, Estrogenrezeptor beta $(\mathrm{ER} \beta)$ und Androgenrezeptor (AR) sowie Auswir- kungen auf die Steroidbiosynthese und damit auf die Östrogen- und Testosteronblutspiegel [18].

Im Vergleich zu PFOA und PFOS sind deren kürzerkettige Derivate weniger gut charakterisiert. In subchronischen und chronischen Tierstudien zeigten z.B. PFHxA oder PFBS vergleichbare adverse Effekte - in Bezug auf die Hepatotoxizität und die Reproduktionstoxizität - wie PFOA und PFOS, jedoch waren hierfür deutlich höhere Dosen erforderlich [11]. Der No-observed-adverse-effect-Level (NOAEL) für die Lebereffekte in der Ratte beträgt für PFOS $0,15 \mathrm{mg} / \mathrm{kg}$ KG pro Tag und für PFOA $0,06 \mathrm{mg} / \mathrm{kg} \mathrm{KG}$ pro Tag $[8,13]$, für PFHxA $10 \mathrm{mg} / \mathrm{kg}$ KG pro Tag [19], für PFBA $6 \mathrm{mg} / \mathrm{kg}$ KG pro Tag [11] und für PFBS $100 \mathrm{mg} / \mathrm{kg} \mathrm{KG}$ pro Tag [11]. Die entsprechenden NOAEL der Substitute der zweiten Generation (GenX, Adona) sind ebenfalls höher als der Wert von PFOA [11, 20]. Molekulare Untersuchungen belegen, dass die kürzerkettigen PFAS die gleichen Wirkmechanismen aufweisen wie PFOA und PFOS, so z. B. hinsichtlich der Aktivierung von PPAR $\alpha$, jedoch sind auch hier deutlich höhere Konzentrationen erforderlich, um vergleichbare Effekte zu erzielen [21, 22].

\section{Exposition}

Der Mensch nimmt PFAS in erster Linie über Trinkwasser, Lebensmittel und in geringerem Ausmaß auch über Hausstaub auf. PFAS sind sowohl in pflanzlichen als auch in tierischen Lebensmitteln nachweisbar. Im größten Teil der Proben der meisten Lebensmittelgruppen liegen 


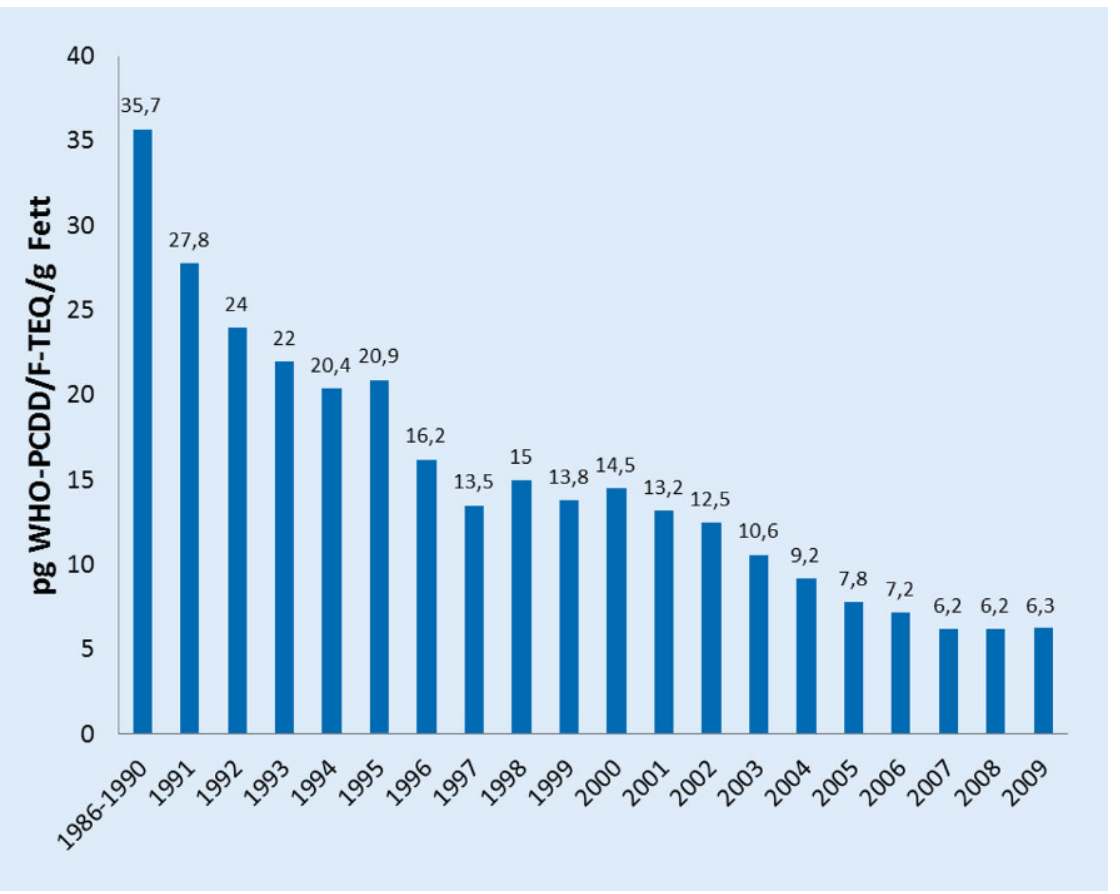

Abb. 3 A Zeitlicher Trend der Gehalte an polychlorierten Dibenzo-p-dioxinen (PCDD) und Dibenzofuranen (PCDF) in Humanmilch aus Deutschland 1986-2009 (dioxinähnliche polychlorierte Biphenyle wurden hier nicht untersucht). TEQ Toxizitätsäquivalent; WHOWeltgesundheitsorganisation (Proben der Dioxindatenbank von Bund und Ländern nach [32])

die Gehalte an PFAS jedoch unterhalb der analytischen Nachweisgrenzen [23].

Schätzungen der Aufnahmemengen an PFAS über Lebensmittel beruhten zunächst auf Gehaltsmessungen in einer relativ schmalen Auswahl an Lebensmitteln, nämlich in erster Linie Trinkwasser und Fisch. Mittlerweile werden Daten zu Gehalten an PFAS in Lebensmitteln in Deutschland im Rahmen des Lebensmittelmonitorings der Bundesländer erhoben. Die Schätzung der täglichen Gesamtexposition beläuft sich laut EFSA (2012) auf maximal 5,2-10,0 ng PFOS und 4,3-7,7 ng PFOA pro kg KG [23]. Die Exposition gegenüber weiteren PFAS liegt nach dieser Expositionsschätzung im Bereich weniger ng/kg KG.

Ergebnisse des von derEU geförderten Forschungsprojekts Perfluorierte organische Verbindungen in unserer Ernährung (PERFOOD) zeigen, dass unterschiedliche Lebensmittelgruppen zur Exposition gegenüberverschiedenen PFASbeitragen [24]. Als relevant sind nach wie vor Trinkwasser, Fisch und Meeresfrüchte anzusehen. Auch weitere tierische Produkte, insbesondere Innereien, und pllanzliche Lebensmittel können messbare Gehalte

\section{Risikocharakterisierung und Fazit}

Die Aufnahmemengen an PFOS und PFOA über Lebensmittel, die die aktuellen Expositionsschätzungen für Verbraucher in Europa bzw. Deutschland ergeben $[23,24,26]$, überschreiten die bisherigen gesundheitlichen Leitwerte der EFSA nicht [13]. Kürzlich abgeleitete Beurteilungswerte für PFOS und PFOA im Blutplasma können hingegen in der Allgemeinbevölkerung überschritten werden [12, 20, 27]. Aus toxikologischer Sicht sind kurzkettige PFAA weniger bedenklich als PFOA und PFOS, da sie zum einen deutlich schneller ausgeschieden werden und zum anderen für die bisher betrachteten toxikologischen Endpunkte bei Nagern ein geringeres toxikologisches Potenzial aufweisen. Da sie jedoch ebenso wie die langkettigen PFAA persistent in der Umwelt sind und zudem besser wasserlöslich und somit mobiler sind, kann eine erhöhte Verbraucherexposition aufgrund von Umwelteinträgen durch Produktion und Endlagerung resultieren, wenn diese Verbindungen in Zukunft verstärkt als Alternativstoffe in der Produktherstellung eingesetzt werden. Die Persistenz der gesamten Stoffgruppe ist darüber hinaus ein Hauptargument, Einträge von PFAS in die Umwelt soweit es geht $\mathrm{zu}$ vermeiden. Das Ziel sollte es sein, fluorfreie und damit echte Alternativen zu PFAS zu entwickeln, damit in Zukunft auf die Produktion von PFAS und die Nutzung von PFAS-haltigen Produkten nach Möglichkeit weltweit verzichtet werden kann.

\section{Polychlorierte Dibenzo-p- dioxine, Dibenzofurane und Biphenyle}

PCDD und PCDF werden auch unter dem Begriff Dioxine zusammengefasst. Dies umfasst 210 verschiedene Einzelsubstanzen, sog. Kongenere, die sich in Anzahl und Stellung der gebundenen Chloratome unterscheiden (• Abb. 2). Die Gruppe der PCDD umfasst 75, die der PCDF 135 Kongenere. PCDD/F sind ubiquitär in der Umwelt vorhanden und in pflanzlichen sowie tierischen Lebensmitteln nachweisbar [28]. Sie 
sind lipophil und reichern sich entlang der Nahrungskette und letztendlich insbesondere im Fett des menschlichen Organismus an. Die biologischen Halbwertzeiten können hier mehrere Jahre betragen [29].

$\mathrm{PCDD} / \mathrm{F}$ entstehen hauptsächlich als Nebenprodukte bei verschiedenen industriellen Prozessen wie z. B. der Müllverbrennung, der Herstellung bestimmter Pestizide oder beim Bleichen von Papier. Natürlicherweise können $\mathrm{PCDD} / \mathrm{F}$ bei Waldbränden oder durch vulkanische Aktivität entstehen [29]. Prozesse und Emissionen, durch die PCDD/F entstehen und in die Umwelt gelangen, sind seit den 1970er-Jahren schrittweise reglementiert und reduziert worden, sodass die Gehalte von PCDD/F in der Umwelt und der Nahrungskette seit den 1980er-Jahren deutlich abnehmen [30, 31]. Die Gehalte in Humanmilch sind ein guter Biomarker für die Exposition der Bevölkerung gegenüber $\mathrm{PCDD} / \mathrm{F}$ und anderen persistenten organischen Kontaminanten. Die durchschnittlichen PCDD/F-Gehalte in Humanmilch in Deutschland aus den Jahren 1986-2009 zeigen einen deutlichen Rückgang der Gehalte etwa um den Faktor 6 (• Abb. 3; [32]). Dieser Trend lässt sich weltweit auch in vielen anderen Ländern beobachten, mit Reduktionsraten von bis zu $5 \%$ pro Jahr [31].

$\mathrm{Zu}$ den PCB gehören 209 verschiedene Kongenere (『Abb. 2); 12 PCB-Kongenere, 4 Non-ortho- und 8 Mono-ortho-PCB werden aufgrund ihrer chemischen und biologischen Eigenschaften zu den dioxinähnlichen PCB gezählt (dl$\mathrm{PCB})$; die übrigen Kongenere bezeichnet man auch als ,non-dioxin-like“ $\mathrm{PCB}$ (ndl-PCB). PCB sind ebenfalls ubiquitär in der Umwelt vorhanden und weisen in ihrem Umweltverhalten große Ähnlichkeiten zu PCDD/F auf. PCB sind in der Vergangenheit zweckbestimmt hergestellt worden, sie wurden vor allem als Kondensatoren- oder Hydraulikflüssigkeiten eingesetzt sowie in Farben und Dichtungsmassen [33, 34].

\section{Gefährdungspotenzial}

$\mathrm{PCDD} / \mathrm{F}$ zeigen tierexperimentell adverse Effekte auf das Nerven-, Immun-,
Hormon- und Reproduktionssystem und sind kanzerogen [28]. Störungen in der Entwicklung dieser Organsysteme wurden in verschiedenen Versuchstierspezies als sensibelste Endpunkte identifiziert [35]. Zwischen verschiedenen Spezies wurden jedoch große qualitative und quantitative Unterschiede bei den Effekten beobachtet. So ist das Meerschweinchen in Bezug auf die Letaldosis mehr als 1000-fach empfindlicher als der Hamster. Dies ist im Wesentlichen auf die unterschiedliche, genetisch vorgegebene Empfindlichkeit und jeweilige biologische Bedeutung des Transkriptionsfaktors Aryl-Hydrocarbon-Rezeptor (AhR) zurückzuführen, dessen Aktivierung die toxischen Wirkungen von PCDD/F vermittelt. Die Bindung eines PCDD/F-Moleküls an den AhR löst eine Kaskade von Reaktionen aus, die letztlich zur Aktivierung bestimmter, bei verschiedenen Spezies teils unterschiedlicher Genabschnitte führt [29].

Toxische Wirkungen von PCDD/F beim Menschen wurden in der Vergangenheit bei Industriearbeitern, nach Unfällen in der chemischen Industrie und bei vorsätzlichen Vergiftungen beobachtet. Klinisch stehen dabei schwerwiegende entzündlich-proliferative Hautveränderungen im Vordergrund, die einer herkömmlichen Akne ähnlich sehen und historisch als Chlor-Akne bezeichnet wurden. Die Bilder der Menschen, insbesondere Kinder, die 1976 nach dem Unfall im norditalienischen Seveso mit Freisetzung von 2,3,7,8-Tetrachlordibenzodioxin (TCDD) um die Welt gingen, haben Dioxine im kollektiven Gedächtnis zum ultimativen Umweltgift werden lassen. Umfangreiche Nachuntersuchungen der betroffenen Bevölkerung über die letzten 40 Jahre haben erfreulicherweise keine oder meist nur grenzwertig signifikante Langzeiteffekte inklusive Krebsentstehung gezeigt. Der bemerkenswerteste Effekt war dabei eine Verschiebung des Geschlechterverhältnisses (mehr Mädchen als Jungen) bei Nachkommen von höher TCDD-exponierten Vätern [36]. Weltweit ist kein Fall einer Dioxinexposition bekannt, die akut zum Tod eines Menschen geführt hat [37].
Dl-PCB weisen in tierexperimentellen Studien ein mit den PCDD/F vergleichbares toxikologisches Profil auf. Dies ist dadurch bedingt, dass die Wirkung aufgrund ihrer planaren Raumstruktur ebenfalls durch Bindung an den AhR vermittelt wird [33]. Studien mit definierten ndl-PCB-Einzelkongeneren im Tiermodell zeigen, dass Leber und Schilddrüse sensible Zielorgane sind. Die Toxizität wird hauptsächlich auf die Induktion von Enzymen der Phasen I und II der Biotransformation sowie auf die Entstehung reaktiver Metabolite zurückgeführt und soll vorwiegend über die Bindung der ndl-PCB an die Kernrezeptoren konstitutiver Androstanrezeptor (CAR) und Pregnan-X-Rezeptor (PXR) vermittelt werden [33].

Die IARC klassifiziert einzelne PCDD/F- und PCB-Kongenere als krebserregend für den Menschen (Gruppe 1). Hierzu zählen TCDD, 2,3,4,7,8-Pentachlorodibenzofuran und $3,3^{\prime}, 4,4^{\prime}, 5$ Pentachlorobiphenyl [38]. Die übrigen $\mathrm{PCDD} / \mathrm{F}-$ Kongenere werden der Gruppe 3 (,not classifiable as to its carcinogenicity to humans") zugeordnet, die übrigen PCB-Kongenere der Gruppe 2A („probably carcinogenic to humans").

Die Weltgesundheitsorganisation (WHO) hat verschiedenen PCDD/Fund dl-PCB-Kongeneren entsprechend ihrer Toxizität Toxizitätsäquivalenzfaktoren (TEF) in Relation zum TCDD zugeordnet, dem toxischsten der verschiedenen Kongenere (TEF = 1; • Tab. 2; [39]). Derzeit sind TEF für 17 PCDD/FKongenere, die mindestens an den Positionen 2,3,7 und 8 chloriert sind, sowie für $12 \mathrm{dl}$-PCB festgelegt. Anhand der TEF lassen sich aus den Gehalten der verschiedenen Einzelkongenere der z. B. in Lebensmitteln enthaltenen PCDD/F und dl-PCB sog. Toxizitätsäquivalente (TEQ) bestimmen, die bei der Risikobewertung sowie der Festlegung von Höchstgehalten in Lebensmitteln zugrunde gelegt werden. Zur Berechnung der TEQ werden die Konzentrationen der einzelnen Kongenere mit dem jeweiligen TEF multipliziert und diese Werte addiert. So lässt sich für die $\mathrm{PCDD} / \mathrm{F}$ Gehalte von Lebensmitteln das WHOPCDD/F-TEQ ermitteln und für dlPCB das WHO-PCB-TEQ. Die Gehalte 
Tab. 2 Toxizitätsäquivalenzfaktoren (TEF) der Weltgesundheitsorganisation (WHO) für die

Risikobewertung von polychlorierten Dibenzo- $p$-dioxinen $(P C D D)$, Dibenzofuranen (PCDF) und dioxinähnlichen polychlorierten Biphenylen (dl-PCB); nach [39]

\begin{tabular}{|c|c|c|c|c|c|}
\hline \multirow[t]{2}{*}{ Kongener } & \multicolumn{2}{|l|}{ TEF } & \multirow[t]{2}{*}{ Kongener } & \multicolumn{2}{|l|}{ TEF } \\
\hline & 1998 & 2005 & & 1998 & 2005 \\
\hline \multicolumn{3}{|l|}{ PCDD } & \multicolumn{3}{|c|}{ Non-ortho-PCB } \\
\hline $2,3,7,8-T C D D$ & 1 & 1 & PCB 77 & 0,0001 & 0,0001 \\
\hline 1,2,3,7,8-PnCDD & 1 & 1 & PCB 81 & 0,0001 & 0,0003 \\
\hline 1,2,3,4,7,8-HxCDD & 0,1 & 0,1 & PCB 126 & 0,1 & 0,1 \\
\hline 1,2,3,6,7,8-HxCDD & 0,1 & 0,1 & PCB 169 & 0,01 & 0,03 \\
\hline 1,2,3,7,8,9-HxCDD & 0,1 & 0,1 & & & \\
\hline $1,2,3,4,6,7,8-\mathrm{HpCDD}$ & 0,01 & 0,01 & & & \\
\hline OCDD & 0,0001 & 0,0003 & & & \\
\hline \multicolumn{3}{|l|}{ PCDF } & \multicolumn{3}{|c|}{ Mono-ortho PCB } \\
\hline 2,3,7,8-TCDF & 0,1 & 0,1 & PCB 105 & 0,0001 & 0,00003 \\
\hline $1,2,3,7,8-P n C D F$ & 0,05 & 0,03 & PCB 114 & 0,0005 & 0,00003 \\
\hline $2,3,4,7,8-\mathrm{PnCDF}$ & 0,5 & 0,3 & PCB 118 & 0,0001 & 0,00003 \\
\hline $1,2,3,4,7,8-\mathrm{HxCDF}$ & 0,1 & 0,1 & PCB 123 & 0,0001 & 0,00003 \\
\hline $1,2,3,6,7,8-\mathrm{HxCDF}$ & 0,1 & 0,1 & PCB 156 & 0,0005 & 0,00003 \\
\hline $1,2,3,7,8,9-\mathrm{HxCDF}$ & 0,1 & 0,1 & PCB 157 & 0,0005 & 0,00003 \\
\hline $2,3,4,6,7,8-\mathrm{HxCDF}$ & 0,1 & 0,1 & PCB 167 & 0,00001 & 0,00003 \\
\hline $1,2,3,4,6,7,8-\mathrm{HpCDF}$ & 0,01 & 0,01 & PCB 189 & 0,0001 & 0,00003 \\
\hline $1,2,3,4,7,8,9-\mathrm{HpCDF}$ & 0,01 & 0,01 & & & \\
\hline OCDF & 0,0001 & 0,0003 & & & \\
\hline \multicolumn{6}{|c|}{$\begin{array}{l}H p C D D \text { Heptachlordibenzodioxin; HpCDF Heptachlordibenzofuran; } H x C D D \text { Hexachlordibenzodi- } \\
\text { oxin; } H x C D F \text { Hexachlordibenzofuran; OCDD Octachlordibenzodioxin; OCDF Octachlordibenzofu- } \\
\text { ran; PeCDD Pentachlordibenzodioxin; PeCDF Pentachlordibenzofuran; TCDD Tetrachlordibenzodi- } \\
\text { oxin; TCDF Tetrachlordibenzofuran }\end{array}$} \\
\hline
\end{tabular}

von $\mathrm{PCDD} / \mathrm{F}$ und dl-PCB fasst man als WHO-PCDD/F-PCB-TEQ zusammen.

Von der WHO wurde als TDI ein Bereich von 1 bis 4 pg WHO-PCDD/FPCB-TEQ pro kg KG aus Toxizitätsstudien abgeleitet [40]. Dabei ist die obere Grenze von 4 pg WHO-PCDD/F-PCBTEQ pro kg KG als provisorische Basis der maximal tolerierbaren Aufnahme zu verstehen. Der untere Wert dokumentiert das Ziel der WHO, die Aufnahme von WHO-PCDD/F-PCB-TEQ beim Menschen auf unter $1 \mathrm{pg} / \mathrm{kg}$ KG pro Tag zu reduzieren. Vom Scientific Committee on Food (SCF) in der EU wurde 2001 eine tolerierbare wöchentliche Aufnahme (TWI) von 14 pg WHO-PCDD/FPCB-TEQ pro kg KG abgeleitet.

\section{Expositionsschätzung und Regulation}

Der Mensch nimmt 90-98\% der Gesamtexposition gegenüber $\mathrm{PCDD} / \mathrm{F}$ und PCB über die Nahrung auf [41], vor- wiegend über Lebensmittel tierischen Ursprungs wie Milch, Fleisch und Fisch [28]. Daher sind in der EU-Höchstgehalte für PCDD/F und PCB nach VO (EG) Nr. 1881/2006 und VO (EG) Nr. 277/2012 insbesondere für verschiedene Lebensund Futtermittel festgelegt, bei deren Überschreitung die Produkte nicht verkehrsfähig sind. Die Höchstgehalte orientieren sich an dem, was aufgrund der Hintergrundbelastung durch PCDD/F oder PCB in der Umwelt unvermeidbar ist. Außerdem sind sog. Auslösewerte oder Aktionsgrenzwerte festgelegt, die unterhalb der Höchstgehalte liegen. Bei deren Überschreitung werden Untersuchungen zur Ermittlung der Kontaminationsquelle eingeleitet und Maßnahmen zur Beschränkung oder Beseitigung der Kontaminationsquelle getroffen. Für Lebensmittel sind jeweils Höchstgehalte für WHO-PCDD/F-TEQ und WHO-PCDD/F-PCB-TEQ festgelegt sowie für die Summe aus 6 ndl-PCB, die als sog. Indikator-PCB zusammen etwa $50 \%$ des Gehalts der 197 ndl-PCBKongenere in der Nahrung ausmachen [28].

Die mittlere tägliche Aufnahme von PCDD/F und dl-PCB über Lebensmittel liegt nach Schätzungen der EFSA in der EU zwischen 0,57 und 2,54 pg WHOPCDD/F-PCB-TEQ pro kg KG [28]. Das Bundesinstitut für Risikobewertung (BfR) geht von einer durchschnittlichen täglichen Aufnahme von 1-2 pg WHOPCDD/F-PCB-TEQ pro $\mathrm{kg}$ KG über die Nahrung für die Bevölkerung in Deutschland aus [42].

Die mittlere tägliche Aufnahme der 6 Indikator-PCB innerhalb der EU wird von der EFSA auf 4,3-5,7 ng pro kg KG geschätzt, für Vielverzehrer liegt die geschätzte Aufnahme bei 7,8-53,7 ng pro kg KG [28]. Der gemeinsame Sachverständigenausschuss für Lebensmittelzusatzstoffe (JECFA) der WHO/Ernährungsund Landwirtschaftsorganisation der Vereinten Nationen (FAO) schätzt die mittlere Exposition der deutschen Bevölkerung gegenüber Indikator-PCB in seinem Bewertungsbericht 2016 auf 9,3-10,8 ng/kg KG pro Tag für Kleinkinder und 5,9-6,2 ng/kg KG pro Tag für Kinder sowie 3,4-3,6 ng/kg KG pro Tag für Erwachsene [33].

Neben der täglichen Aufnahme von $\mathrm{PCDD} / \mathrm{F}$ und $\mathrm{PCB}$ über die Nahrung ist auch die Körperlast des Menschen ein wichtiger Parameter für die Exposition. Unter Körperlast oder „body burden“ versteht man die Menge an PCDD/F und PCB, die ein Mensch bei der täglichen Aufnahme bis zu einem bestimmten Zeitpunkt seines Lebens in seinem Körper angereichert hat. Diese unterscheidet sich individuell abhängig von Alter, Verzehrgewohnheiten, Beruf und Lebensraum einer Person und kann - da sich die Verbindungen gleichmäßig im Körperfett verteilen - durch eine Analyse von Blutfett, Körperfett oder Humanmilch (• Abb. 3) bestimmt werden.

\section{Risikocharakterisierung und Fazit}

Die geschätzte mittlere Aufnahme der Bevölkerung von 1-2 pg WHO-PCDD/ F-PCB-TEQ pro kg KG pro Tag über die Nahrung liegt im Bereich gesundheitsbezogener Leitwerte wie des TDI von 1-4 pg 


\begin{tabular}{|c|c|c|c|}
\hline \multirow[t]{2}{*}{ Priority-PAK } & \multirow[t]{2}{*}{ Wirkungsäquivalente } & \multicolumn{2}{|c|}{ Indikator-PAK } \\
\hline & & PAK4 ${ }^{\mathrm{a}}$ & PAK $^{\mathrm{b}}$ \\
\hline Benzo[a]pyren & 1 & $x$ & $x$ \\
\hline Benzo[a]anthracen & 0,1 & $x$ & $x$ \\
\hline Benzo[b]fluoranthen & 0,1 & $x$ & $x$ \\
\hline Chrysen & 0,01 & $x$ & $x$ \\
\hline Benzo[ghi]perylen & - & & $\mathrm{x}$ \\
\hline Benzo[k]fluoranthen & 0,1 & & $x$ \\
\hline Dibenzo[a,h]anthracen & 1 & & $x$ \\
\hline Indeno[1,2,3-cd]pyren & 0,1 & & $\mathrm{x}$ \\
\hline Benzo[j]fluoranthen & 0,1 & & \\
\hline Cyclopenta[cd]pyren & 0,1 & & \\
\hline Dibenzo[a,e]pyren & 1 & & \\
\hline Dibenzo $[a, h]$ pyren & 10 & & \\
\hline Dibenzo[a,i]pyren & 10 & & \\
\hline Dibenzo[ $[a, /]$ pyren & 10 & & \\
\hline 5-Methylchrysen & - & & \\
\hline Benzo[c]fluoren & - & & \\
\hline \multicolumn{4}{|c|}{ 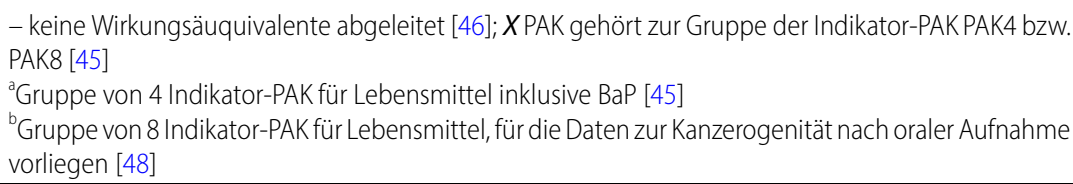 } \\
\hline
\end{tabular}

der WHO oder des TWI des SCF von 14 pg WHO-PCDD/F-PCB-TEQ pro kg KG. Aufgrund der sehr langen Halbwertzeiten von $\mathrm{PCDD} / \mathrm{F}$ und $\mathrm{dl}-\mathrm{PCB}$ sind diese täglichen Aufnahmedosen klein gegenüber der im Lauf des Lebens akkumulierten Körperlast, die letztlich für eine mögliche gesundheitliche Wirkung verantwortlich ist. Deshalb sind bei kurzbis mittelfristigen leichten Überschreitungen der gesundheitsbezogenen Leitwerte durch die Aufnahme von WHOPCDD/F-PCB-TEQ über die Nahrung keine negativen Auswirkungen auf die menschliche Gesundheit zu erwarten.

Für ndl-PCB sind bisher keine gesundheitsbezogenen Leitwerte abgeleitet worden. In einem aktuellen Bewertungsbericht kommt das JECFA unter Anwendung des Margin-of-Exposure-Konzepts [43] und Berücksichtigung der Körperlast zu der Einschätzung, dass die Exposition gegenüber ndl-PCB über die Nahrung für Erwachsene und Kinder wahrscheinlich nicht bedenklich ist. Gestillte Säuglinge, als besonders exponierte Bevölkerungsgruppe, schließt diese Einschätzung nicht mit ein, die Vorteile des Stillens überwiegen jedoch mögliche Ri-

\section{Gefährdungspotenzial, Exposition und Regulation}

Die Exposition der Bevölkerung gegenüber PAK erfolgt hauptsächlich über die Atemluft inklusive Tabakrauch und über Lebensmittel. An Partikel gebunden werden PAK über die Luft weite Strecken transportiert und gelangen so, z. B. durch Adhäsion an pflanzlichen Lebensmitteln, auch in die Nahrungskette. Außerdem entstehen PAK im Lebensmittel während thermischer $\mathrm{Zu}$ bereitungsprozesse wie dem Grillen, Rösten und Räuchern. Sie sind daher in verschiedenen Lebensmittelgruppen wie Gemüse, Obst, pflanzlichen Fetten, Fisch und Meeresfrüchten, geräucherten und gegrillten Speisen, Kakao und Schokolade nachweisbar [45].

PAK sind fettlöslich und verteilen sich nach inhalativer, dermaler und oraler Aufnahme insbesondere in fettreichen Gewebeanteilen. Einige Verbindungen können von verschiedenen Enzymsystemen zu hochreaktiven, genotoxischen Verbindungen, z. B. Dihydrodiolepoxiden, metabolisiert werden und induzieren die Expression der Enzyme, die zu ihrer Bioaktivierung beitragen, zum Teil selbst. Ihr genotoxisches kanzerogenes Potenzial steht bei der gesundheitlichen Bewertung von PAK im Vordergrund. Ein gesundheitlicher Leitwert im Sinn einer tolerierbaren täglichen Aufnahmemenge kann für PAK daher nicht abgeleitet werden. Das kanzerogene Wirkpotenzial der Einzelverbindungen ist unterschiedlich hoch und korreliert mit dem Vorhandensein bestimmter Strukturmerkmale, lässt sich allerdings nicht allein daraus ableiten [46, 47]. Aus vergleichenden Untersuchungen zum kanzerogenen Potenzial von Benzo[a]pyren (BaP) $\mathrm{zu}$ anderen PAK-Einzelsubstanzen oder Gemischen in verschiedenen Testmodellen wurden Wirkungsäquivalenzfaktoren abgeleitet (• Tab. 3; [46]). Im Unterschied zu den PCDD/F und dlPCB konnte sich dieses System bisher nicht für die gesundheitliche Bewertung von PAK in Lebensmitteln durch die EFSA durchsetzen.

Stattdessen wurde für die PAK ein sog. Stellvertretersystem zur Bewertung der Mischungsexposition in Lebensmit- 
teln etabliert, bei dem zunächst $\mathrm{BaP}$ als Leitsubstanz stellvertretend für die gesamte Gruppe im Sinn eines Markers für das Vorkommen und die Toxizität von PAK-Gemischen betrachtet wurde.

Inzwischen wurde das Spektrum der Stellvertretersubstanzen um 3 Stoffe erweitert (PAK4), die wie $\mathrm{BaP}$ zu einer Gruppe von 16 potenziell genotoxischen und kanzerogenen sog. Priority-PAK gehören $[45,49]$, von denen für 8 PAK Daten zur Kanzerogenität nach oraler Aufnahme vorliegen (PAK8; - Tab. 3; [48]). In einer groß angelegten Auswertung von Analysen in Lebensmitteln zeigten sich diese 4 PAK als geeignete Indikatoren für das Vorkommen von PAK in Lebensmitteln, da mit ihnen $94 \%$ der Proben als PAK-haltig detektiert wurden, in denen Stoffe der 16 Priority-PAK enthalten waren [45]. Die unterschiedlichen toxischen Potenziale der individuellen PAK bleiben dabei allerdings unberücksichtigt. Eine Weiterentwicklung eines Bewertungssystems, das auf Wirkungsäquivalenzfaktoren beruht, wäre hierfür erforderlich, insbesondere weil auch Priority-PAK mit ausgesprochen hohen Faktoren, wie bestimmte Dibenzopyrene, nicht in der Gruppe der 4 Indikator-PAK enthalten sind (-Tab.3). Die Expositionsschätzung der EFSA (2008) ergab für die europäische Bevölkerung im Median eine Aufnahme von 19,5 ng PAK4/kg KG pro Tag bei mittleren Verzehrmengen und 34,5 ng PAK4/kg KG pro Tag bei hohen Verzehrmengen (95. Perzentil; [45]). Den größten Anteil an der PAK-Exposition über Lebensmittel haben Getreide und Getreideprodukte sowie Meeresfrüchte.

Da es sich bei PAK um genotoxische Kanzerogene handelt, gilt das Minimierungsgebot; Höchstgehalte für diese Stoffgruppe sind so niedrig wie nach aktuellem Stand der Technik erreichbar festzulegen. Die Auswahl an Indikatorverbindungen sollte kontinuierlich an Erkenntnisse zum kanzerogenen Potenzial der Einzelverbindungen und ihr Vorkommen in Lebensmitteln angepasst werden [50].

\section{Fazit}

Die Persistenz der diskutierten Stoffgruppen in der Umwelt führt dazu, dass eine Exposition der Bevölkerung über lange Zeiträume bestehen bleibt, auch wenn Einträge in die Umwelt minimiert werden. Bei Verbindungen mit langen Halbwertzeiten kommt hinzu, dass auch bei geringen Gehalten in Lebensmitteln eine zunehmende Körperlast resultieren kann, wenn die Lebensmittel regelmäßig über einen längeren Zeitraum verzehrt werden.

Das Beispiel der PCDD/F zeigt eindrücklich, dass eine effektive Regulation zu einer langfristigen Abnahme der Gehalte in der Umwelt und somit auch im menschlichen Organismus führen kann. Auch wenn sich die Exposition über Lebensmittel dadurch maßgeblich vermindert hat, liegt sie gegenwärtig dennoch im Bereich gesundheitlicher Leitwerte und eine Weiterführung der Minimierungsmaßnahmen ist weiterhin erforderlich. Eine ähnliche Entwicklung lässt sich auch für die Stoffgruppe der PCB beobachten. Zur Erfassung der Höhe des toxischen Potenzials einer Mischexposition ist für PCDD/F und dlPCB ein TEF-System etabliert. Für andere Stoffgruppen wie PAK und PFAS wird ein solches System bislang weder bei der gesundheitlichen Bewertung noch bei der Regulation verwendet. Das für die gesundheitliche Bewertung von PAK etablierte Stellvertretersystem berücksichtigt keine Informationen zur Höhe des kanzerogenen Potenzials der Einzelverbindungen. Für die große Gruppe der PFAS können weitere Untersuchungen zu den molekularen Mechanismen der toxischen Wirkungen die Datenbasis zur relativen Höhe des toxischen Potenzials von Einzelverbindungen verbreitern. Gesundheitliche Beurteilungswerte für PFAS liegen bislang nur für 2 Verbindungen der Stoffgruppe, PFOS und PFOA, vor. Aktuell wird für PFAS die Reevaluierung der bisher zur Bewertung herangezogenen TDI-Werte diskutiert, da mittlerweile eine breite Datenbasis aus epidemiologischen Studien vorhanden ist und neue Erkenntnisse zu mehreren toxikologischen Endpunkten, insbesondere zur Immuntoxizität, vorliegen. Eine besondere Herausforderung für die gesundheitliche Bewertung von PFAS in Lebensmitteln besteht darin, die Be- deutung anderer PFAS-Verbindungen inklusive sog. Vorläuferstoffe sowohl bezüglich der Exposition als auch der Toxizität zu klären und weitere Beurteilungswerte abzuleiten.

\section{Korrespondenzadresse}

\section{Dr. U. Pabel}

Bundesinstitut für Risikobewertung

Berlin, Deutschland

ulrike.pabel@bfr.bund.de

\section{Einhaltung ethischer Richtlinien}

Interessenkonflikt. U. Pabel, T. Buhrke, K. Abraham, T. Nölke, M. Gehling, A. Lampen, M. LahrssenWiederholt und R. Wittkowski geben an, dass kein Interessenkonflikt besteht.

Dieser Beitrag beinhaltet keine von den Autoren durchgeführten Studien an Menschen oder Tieren.

Open Access. Dieser Artikel wird unter der Creative Commons Namensnennung 4.0 International Lizenz (http://creativecommons.org/licenses/by/4.0/deed. de) veröffentlicht, welche die Nutzung, Vervielfältigung, Bearbeitung, Verbreitung und Wiedergabe in jeglichem Medium und Format erlaubt, sofern Sie den/die ursprünglichen Autor(en) und die Quelle ordnungsgemäßnennen, einen Linkzur Creative Commons Lizenz beifügen und angeben, ob Änderungen vorgenommen wurden.

\section{Literatur}

1. Giesy J, Kannan K (2002) Perfluorochemical surfactants in the environment. Environ Sci Technol 36:146A-152A

2. Hölzer J, Midasch O, Rauchfuss K, Kraft M, Reupert $R$ et al (2008) Biomonitoring of perfluorinated compounds in children and adults exposed to perfluorooctanoate-contaminated drinking water. Environ Health Perspect 116:651-658

3. Buck RC, Franklin J, Berger U et al (2011) Perfluoroalkyl and polyfluoroalkyl substances in the environment: terminology, classification, and origins. Integr Environ Assess Manag 7:513-541

4. Rand AA, Mabury SA (2017) Is there a human health risk associated with indirect exposure to perfluoroalkyl carboxylates (PFCAs)? Toxicology 375:28-36

5. Lau C (2015) Perfluorinated compounds: an overview. In: DeWitt JC (Hrsg) Toxicological effects of perfluoroalkyl and polyfluoroalkyl substances. Humana Press Springer, Switzerland, S1-21

6. Numata J, Kowalczyk J, Adolphs J, Ehlers S, Schafft $\mathrm{H}$ et al (2014) Toxicokinetics of seven perfluoroalkyl sulfonic and carboxylic acids in pigs fed a contaminated diet. J Agric Food Chem 62:6861-6870

7. Zhang Y, Beesoon S, Zhu L, Martin JW (2013) Biomonitoring of perfluoroalkyl acids in human urine and estimates of biological half-life. Environ Sci Technol 47:10619-10627 
8. ATSDR(2015)Draft toxicological profile forperfluoroalkyls. https://www.atsdr.cdc.gov/toxprofiles/ tp200.pdf. Zugegriffen: 12. Jan. 2017

9. NTP (2016) Monograph on Immunotoxicity Associated with Exposure to Perfluorooctanoic Acid (PFOA) and Perfluorooctane Sulfonate (PFOS). http://ntp.niehs.nih.gov/ntp/ohat/pfoa pfos/pfoa_pfosmonograph_508.pdf.Zugegriffen: 12. Jan. 2017

10. Lau C, Thibodeaux JR, Hanson RG et al (2006) Effects of perfluorooctanoic acid exposure during pregnancy in the mouse. Toxicol Sci 90:510-518

11. Bull S, Burnett K, Vassaux K, Ashdown L, Brown T, Rushton L (2014) Extensive literature search and provision of summaries of studies related to the oral toxicity of perfluoroalkylated substances (PFASs), their precursors and potential replacements in experimental animals and humans. EFSA supporting publication. http://onlinelibrary. wiley.com/doi/10.2903/sp.efsa.2014.EN-572/pdf. Zugegriffen: 12. Jan. 2017

12. UBA (2016) HBM-I-Werte für Perfluoroctansäure (PFOA) und Perfluoroctansulfonsäure (PFOS) in Blutplasma, Stellungnahme der Komission Human-Biomonitoring des Umweltbundesamtes. Bundesgesundheitsblatt Gesundheitsforschung Gesundheitsschutz 59:1362-1363

13. EFSA (2008) Opinion of the scientific panel on contaminantsin the food chain on perfluorooctane sulfonate (PFOS), perfluorooctanoic acid (PFOA) and their salts. EFSA J 653:1-131. doi:10.2903/j. efsa.2008.653/full

14. US-EPA (2016) Drinking water health advisory for perfluorooctane sulfonate (PFOS). EPA document number: 822-R-16-004. https://www.epa.gov/ sites/production/files/2016-05/documents/pfos health_advisory_final_508.pdf. Zugegriffen: 12. Jan. 2017

15. US-EPA (2016) Drinking water health advisory for perfluorooctanoic acid (PFOA). EPA document number: 822-R-16-005. https://www.epa. gov/sites/production/files/2016-05/documents/ pfoa_health_advisory_final_508.pdf. Zugegriffen: 12. Jan. 2017

16. Buhrke T, Krüger E, Pevny S, Rößler $M$, Bitter $K$, Lampen A (2015) Perfluorooctanoic acid (PFOA) affects distinct molecular signalling pathways in human primary hepatocytes. Toxicology 333:53-62

17. Scharmach $E$, Buhrke $T$, Lichtenstein $D$, Lampen $A$ (2012) Perfluorooctanoic acid affects the activity of the hepatocyte nuclear factor 4 alpha (HNF4a). Toxicol Lett 212:106-112

18. Kjeldsen LS, Bonefeld-Jørgensen EC (2013) Perfluorinated compounds affect the function of sex hormone receptors. Environ Sci Pollut Res Int 20:8031-8044

19. Chengelis CP, Kirkpatricka JB, Radovskya A, Shinoharab M (2009) A 90day repeated dose oral (gavage) toxicity study of perfluorohexanoic acid (PFHxA) in rats (with functional observational battery and motor activity determinations). Reprod Toxicol 27:342-351

20. Fromme H, Wöckner M, Roscher E, Völkel W (2016) ADONA and perfluoroalkylated substances in plasma samples of German blood donors living in South German. Int J Hyg Environ Health. doi:10. 1016/j.ijheh.2016.12.014

21. Buhrke T, Kibellus A, Lampen A (2013) In vitro toxicological characterization of perfluorinated carboxylic acids with different carbon chain lengths. Toxicol Lett 218:97-104

22. Wolf CJ, Schmid JE, Lau C, Abbott BD (2012) Activation of mouse and human peroxisome proliferator-activated receptor-alpha (PPARa) by perfluoroalkyl acids (PFAAs): Further investigation of C4-C12 compounds (2012). Reprod Toxicol 33:546-551

23. EFSA (2012) Perfluoroalkylated substances in food: occurrence and dietary exposure. EFSA J 10:2743-2789. doi:10.2903/j.efsa.2012.2743/epdf

24. Klenow S, Heinemeyer G, Brambilla G, Dellatte E, Herzke D, de Voogt P (2013) Dietary exposure to selected perfluoroalkyl acids (PFAAs) in four European regions. Food Addit Contam Part AChem Anal Control Expo Risk Assess 30:2141-2151

25. Kowalczyk J, Ehlers S, Oberhausen A, Tischer M, Fürst $P$ et al (2013) Absorption, distribution, and milk secretion of the perfluoroalkyl acids PFBS, PFHxS, PFOS, and PFOA by dairy cows fed naturally contaminated feed. J Agric Food Chem 61:2903-2912

26. BfR (2008) Gesundheitliche Risiken durch PFOS und PFOA in Lebensmitteln sind nach dem derzeitigen wissenschaftlichen Kenntnisstand unwahrscheinlich. Stellungnahme 004/2009 des BfR vom 11. September 2008. http://www. bfr.bund.de/cm $/ 343 / g e s u n d h e i t l i c h e \_r i s i k e n$ durch_pfos_und_pfoa_in_lebensmitteln.pdf. Zugegriffen: 12. Jan. 2017

27. Schröter-Kermani C, Müller J, Jürling H, Conrad A, Schulte C (2013) Retrospective monitoring of perfluorocarboxylates and perfluorosulfonates in human plasma archived by the German Environmental Specimen Bank. Int J Hyg Environ Health 216:633-640

28. EFSA (2012) Update of the monitoring of levels of dioxins and PCBs in food and feed. EFSA J 10:2832. doi:10.2903/j.efsa.2012.2832/pdf

29. Schecter A, Birnbaum L, Ryan JJ, Constable JD (2006) Dioxins: an overview. Environ Res 101:419-428

30. Schwartz M, Lindtner O, Blume K, Heinemeyer G, Schneider K (2014) Dioxin and dl-PCB exposure from food: the German LExUKon project. Food Addit Contam Part A Chem Anal Control Expo Risk Assess 31:688-702

31. WHO Europe (2009) Persistent Organic Pollutants in Human Milk. Fact Sheet 4.3 Code RPG4_Food_Ex2. European Environment and Health Information System ENHIS. http:// www.euro.who.int/ data/assets/pdf file/0003/ 97032/4.3.-Persistant-Organic-PollutantsmEDITED_layouted_V2.pdf. Zugegriffen: 23. Jan. 2017

32. BfR (2011) Frauenmilch: Dioxingehalte sinken kontinuierlich. Information Nr. 011/2011 des BfR vom 23.03.2011. http://www.bfr.bund. $\mathrm{de} / \mathrm{cm} / 343 /$ frauenmilch_dioxingehalte_sinken_ kontinuierlich.pdf.Zugegriffen: 12. Jan. 2017

33. WHO (2016) Safety evaluation of certain food additives and contaminants. Supplement 1: Nondioxin-like polychlorinated biphenyls. Eightieth meeting of the Joint FAO/WHO Expert Committe on Food Additives (JECFA). WHO Food Additives Series 71-S1. http://apps.who.int/iris/bitstream/ 10665/246225/1/9789241661713-eng.pdf. Zugegriffen:23. Jan. 2017

34. Fromme $H$, Hilger B, Albrecht M, Gries W, Leng G, Völkel W (2016) Occurrence of chlorinated and brominated dioxins/furans, PCBs, and brominated flame retardants in blood of German adults. Int J Hyg Environ Health 219:380-388

35. Birnbaum LS, Tuomisto J (2000) Non-carcinogenic effects of TCDD in animals. Food Addit Contam 17:275-288

36. Mocarelli P, Gerthoux PM, Ferrari E et al (2000) Paternal concentrations of dioxin and sex ratio of offspring. Lancet 355:1858-1863
37. Martinez JM, DeVito MJ, Birnbaum LS, Walker NJ (2003) Toxicology of dioxins and dioxinlike compounds. In: Schecter A, Gasiewicz TA (Hrsg) Dioxins and health. Wiley-Interscience, Hoboken NJ, S 137-142

38. IARC (2012) 2,3,7,8-TetrachlorodibenzoparaDioxin, 2,3,4,7,8-Pentachlorodibenzofuran, and $3,3^{\prime}, 4,4^{\prime}, 5$-Pentachlorobiohenyl. Monographs on the Evaluation of Carcinogenic Risks to Humans, No. 100F. http://monographs.iarc.fr/ ENG/Monographs/vol100F/mono100F-27.pdf. Zugegriffen: 12. Jan. 2017

39. Van den Berg M, Birnbaum LS, Denison $M$ et al (2006) The 2005 World Health Organization re-evaluation of human and mammalian toxic equivalency factors for dioxins and dioxin-like compounds. Toxicol Sci 93:223-241

40. WHO (2000) Consultation on assessment of the health risk of dioxins; re-evaluation of the tolerable daily intake (TDI): Executive Summary. Food Addit Contam 17:223-240

41. Malisch R, Kotz A (2014) Dioxins and PCBs in feed and food - review from European perspective. Sci Total Environ 491-492:2-10

42. BfR (2012) Fragen und Antworten zu Dioxinen und PCB in Lebensmitteln. Aktualisierte FAQ des BfR vom 13. April 2012. http://www.bfr.bund. $\mathrm{de} / \mathrm{cm} / 343 /$ fragen-und-antworten-zu-dioxinenund-pcb-in-lebensmitteln.pdf. Zugegriffen: 12. Januar 2017

43. EFSA (2005) Opinion of the Scientific Committee on a request from EFSA related to a harmonised approach for risk assessment of substances which are both Genotoxic and carcinogenic. EFSA J 282:1-31.doi:10.2903/j.efsa.2005.282/pdf

44. WHO (2002) Evaluation of certain food additives and contaminants. Fifty-seventh report of the Joint FAO/WHO Expert Committee on Food Additives (JECFA). WHO Technical Report Series 909. http://apps.who.int/iris/bitstream/10665/ 42578/1/WHO_TRS 909.pdf.Zugegriffen: 12. Jan. 2017

45. EFSA (2008) Polycyclic aromatic hydrocarbons in food. EFSA J 724:1-114

46. DFG (2008) Polycyclische Aromatische Kohlenwasserstoffe (PAH). MAK Value Documentation. The MAK Collection for Occupational Health and Safety, 45. Lieferung 1-210. http://onlinelibrary.wiley.com/doi/10.1002/ 3527600418.mb0223orgd0045/pdf. Zugegriffen: 12. Jan. 2017

47. Flesher JW, Lehner AF (2017) Structure, function and carcinogenicity of metabolites of methylated and non-methylated polycyclic aromatic hydrocarbons: a comprehensive review. Toxicol Mech Methods 26:151-179

48. Culp SJ, Gaylor DW, Sheldon WG, Goldstein LS, Beland FA (1998) A comparison of the tumours induced by coal tar and benzo[a]pyrene in a 2-year bioassay. Carcinogenesis 19:117-124

49. EC (2002) Opinion of the Scientific Committee on Food on the risks to human health of Polycyclic Aromatic Hydrocarbons in food. Scientific Committee on Food SCF/CS/CNTM/PAH/29 Final. https:// ec.europa.eu/food/sites/food/files/safety/docs/ sci-com_scf_out153_en.pdf.Zugegriffen:12.Jan. 2017

50. BfR (2009) Markersubstanzen für polyzyklische aromatische Kohlenwasserstoffe (PAK) zur Lebens mittelüberwachung. Stellungnahme Nr. 003/2010 des BfR vom 02. Oktober 2010. http://www.bfr. bund.de/cm/343/markersubstanzen_fuer_pak zur_lebensmittelueberwachung.pdf.Zugegriffen: 12. Jan. 2017 